\title{
Linx
}

Revue des linguistes de l'université Paris X Nanterre

$73 \mid 2016$

Énonciation et marques d'oralité dans l'évolution du français

\section{Qui dit amoureux, dit triste : syntaxe et sémantique du marqueur Qui dit $X$, dit $Y$ du français préclassique au français contemporain}

\section{Sonia Gómez-Jordana Ferary}

\section{OpenEdition}

\section{Journals}

Édition électronique

URL : https://journals.openedition.org/linx/1626

DOI : 10.4000/linx.1626

ISSN : 2118-9692

Éditeur

Presses universitaires de Paris Nanterre

Édition imprimée

Date de publication : 1 septembre 2016

Pagination : 39-64

ISSN : 0246-8743

Référence électronique

Sonia Gómez-Jordana Ferary, «Qui dit amoureux, dit triste : syntaxe et sémantique du marqueur Qui dit $X$, dit $Y$ du français préclassique au français contemporain », Linx [En ligne], 73 | 2016, mis en ligne le 01 mars 2017, consulté le 21 septembre 2021. URL : http://journals.openedition.org/linx/1626 ; DOI https://doi.org/10.4000/linx.1626 


\title{
Qui dit amoureux, dit triste : syntaxe et sémantique du marqueur Qui dit $\mathrm{X}$, dit $\mathrm{Y}$ du français préclassique au français contemporain
}

\begin{abstract}
Résumé $^{1}$
Le but de notre article est de rendre compte de l'évolution syntaxique et sémantique d'un marqueur qui, à notre connaissance, n'a pas attiré l'attention des linguistes : Qui dit X, dit Y. L'étude syntaxique du marqueur en diachronie permet de vérifier une évolution dans sa structure. Ainsi, le marqueur non seulement présente une forme beaucoup plus complexe en français préclassique et classique qu'en français contemporain, mais en outre le nombre de variantes est également beaucoup plus nombreux en français classique que de nos jours. À la suite de l'étude syntaxique, nous proposons une analyse sémantique du marqueur où il apparaît que le sens de celui-ci évolue également au cours des siècles. Ainsi, la locution véhicule en français préclassique et classique une relation définitionnelle entre les segments $\mathrm{X}$ et $\mathrm{Y}$, alors qu'en français contemporain, la relation devient stéréotypique entre $\mathrm{X}$ et $\mathrm{Y}$, où $\mathrm{Y}$ est présenté comme le prototype de $\mathrm{X}$.
\end{abstract}

Abstract

I describe here the syntactic and semantic evolution of a discourse marker which, to my knowledge, has not drawn the linguists' attention: Qui dit X, dit Y. The historical syntactic study of the marker reveals an evolution. The marker presents a more complex structure in Preclassical and Classical French than in Contemporary French. Moreover, the number of possible variants is more important in Classical French than nowadays. After the syntactic study, I will present a semantic analysis of the marker, showing that its meaning evolves through time. Thus, the phrase shows a definitional relation in Preclassical and Classical French between the segments X and Y, whereas in Contemporary French the relation between $\mathrm{X}$ and $\mathrm{Y}$ becomes stereotypical. $\mathrm{Y}$ is shown as the prototype of $\mathrm{X}$.

$1 \quad$ Cet article a été réalisé dans le cadre du projet de recherche FFi2013-41355-P « Marqueurs pragmatiques et oralité en linguistique historique du français » du Ministerio de Economía y Competitividad, Espagne, (Plan Estatal i+D+i 2013-16). 
Nous nous proposons dans cet article d'étudier l'évolution d'un marqueur du discours Qui dit X, dit Y, d'un point de vue syntaxique et sémantique en diachronie, depuis le français préclassique et classique, où la locution présente une grande variété de structures, jusqu'au français contemporain.

Le cadre théorique sera d'une part celui de la pragmatique historique. Nous nous servirons également de la théorie des stéréotypes, développée par Jean-Claude Anscombre depuis la moitié des années 1990. Notre étude relève également des travaux sur les marqueurs médiatifs telles que Guéntcheva (1996) ou Dendale et Tasmowski (1994), et de la théorie de la polyphonie telle qu'elle est développée dans Ducrot (1984). Le corpus employé provient principalement de la base de données Frantext.

\section{PROPRIETES SYNTAXIQUES ET DISTRIBUTIONNELLES}

\subsection{Propriétés syntaxiques de la locution en français préclassique et classique}

Ni les dictionnaires ni les grammaires ne semblent s'intéresser à la locution verbale Qui dit X, dit Y. Grevisse (1993) aborde plusieurs locutions en dire, telles que je te dirai quoi, comme qui dirait, à ce qu'il dit, mais ne signale pas celle-ci. De même, Riegel et alii (1994) ne font pas référence à la locution. Elle est également absente des grammaires historiques, telles que Gougenheim (1984), Grammaire française du seizième siècle, Spillebout (1985=2007), Grammaire de la langue française du xvii ${ }^{\text {e }}$ siècle, Fournier (1998), Grammaire du français classique, ou Lardon et Thomine (2009), Grammaire du français de la Renaissance, alors qu'elle est fort employée aux XVII et XVIII ${ }^{\mathrm{e}}$ siècles. Les dictionnaires ne mentionnent pas la locution. C'est le cas de dictionnaires historiques tels que Jean Nicot (1621) Le Thrésor de la langue française, ou le Dictionnaire Universel de Furetière (1690), où la structure en Qui dit X, dit Y est absente. Rey (2012), Le Dictionnaire historique de la langue française, n'aborde pas non plus la locution.

Le Dictionnaire de la langue française de Littré (1872) cite dans l'acception du verbe dire une occurrence où apparait la locution, mais alors qu'il explique toutes les autres locutions, pour celle-ci il ne fait que donner un exemple, à savoir :

(1) Car afin que l'on le sache,

C'est Sillery qui s'attache

A vouloir que de nouveau,

Sire Loup, Sire Corbeau

Chez moi se parlent en rime.

Qui dit Sillery, dit tout ;

Peu de gens en leur estime

Lui refusent le haut bout.

La Fontaine (1674), Tircis et Amarante, Fable VIII, 13.

Quant au dictionnaire Le Robert (2001), il signale la locution parmi les formules familières qui servent à employer telles formes linguistiques pour exprimer quelque chose : " "Qui dit (emploie l'expression) fils à papa, dit (exprime) jeune homme gâté, paresseux", "Qui dit froid écrivain, dit détestable auteur"

(Boileau). »

La première occurrence dans Frantext date du début du xvii ${ }^{e}$ siècle, en 1607.

(2) (...) c'est là vostre soulas, duquel vous ne vous pouvez saouler : c'est là vostre recreation vrayement royale, et digne d'une ame royale : qui dit royale, dit tout, ainsi que respondit Porus au grand Alexandre. 
Scipion Dupleix, La Logique ou l'Art de discourir et raisonner, 1607, p.8.

Auparavant, au $\mathrm{xvi}^{\mathrm{e}}$ siècle, existe la structure syntaxique en Qui dit $\mathrm{X}$, il dit $\mathrm{Y}$, mais elle n'a pas le même sens. Alors que la locution possède un sens générique du type de $A$ chaque fois que l'on dit $X$, c'est comme si l'on disait $Y$, l'exemple de Du Bellay ne vérifie pas cette lecture générique. Dans ce cas, le pronom qui est paraphrasable par celui qui et la lecture est spécifique :

(3) Qui dit que tu es sage, il dit la vérité Du Bellay, Les Regrets, 1558.

$\mathrm{Au} \mathrm{Xv}^{\mathrm{e}}$ siècle, Littré cite une structure qui pourrait avoir un rapport avec Qui dit X, dit Y :

(4) Onques homme sage ne monta sur asne, pour l'onneur de nostre Seigneur, qui dessus monta, mais tresbien surcheval, car qui chiet de l'asne il dist crieve, et qui chiet du cheval il dit liève.

Glose. Sur cest article se puet faire un argument, car quant Joseph mena la Vierge Marie en Egypte, elle monta sur un asne, et toutesfois elle n'en eut nul grief. (...)

Fouquart de Cambray, (1480), Les Evangiles des quenouilles, p. 34 (édition de 1855, Paris), cité dans Littré.

[Tomber de l'âne signifie crever, (si on tombe de l'âne, on meurt) tomber du cheval signifie se lever (si on tombe du cheval, on se lève).]

La locution Qui dit X, dit $\mathrm{Y}$ a comme particularité de présenter le pronom relatif qui sans antécédent, trait que partagent de nombreux proverbes - Qui dort dîne, Qui veut tuer son chien, l'accuse de la rage... Le pronom relatif sans antécédent possède une lecture implicative en français contemporain, paraphrasable en Si on, comme l'a démontré entre autres Riegel (1987). La valeur du pronom sans antécédent a varié à d'autres stades de la langue française. L'étude de Bertrand (2003 : 387) montre comment le pronom possédait en ancien français aussi bien la valeur générique paraphrasable par si on que celle en celui qui. L'auteur signale que la frontière entre les deux lectures est infime dans des exemples tels que Plaindre se doit qui est batuz, paraphrasable aussi bien par Celui qui est battu doit se plaindre que par Si l'on est battu, on doit se plaindre. D'autres auteurs, comme Ménard (1994), rappellent la valeur générique du pronom en ancien et moyen français, ce que l'on peut vérifier dans des exemples comme Qui a marastre a diable en l'astre (Si l'on a une belle-mère, on a un diable au foyer), Ménard (1994: 79). Le pronom relatif qui en emploi autarcique avec valeur indéfinie - tout homme qui - subsiste, comme le rappellent Lardon et Thomine (2009:164) jusqu'au XVII ${ }^{\mathrm{e}}$ siècle $^{2}$. Cependant, il est considéré comme un vestige en français préclassique et classique. C'est ce que montre Gougenheim (1974 : 97) dans sa grammaire du xvi ${ }^{\mathrm{e}}$ siècle par le biais du proverbe Tout vient à point qui sait attendre, où le pronom équivaut à si on. De nos jours, le proverbe présente la préposition à - Tout vient à point à qui sait attendre - d'où la lecture en celui qui : Tout vient à point à celui qui sait attendre ${ }^{3}$. Quoi qu'il en soit, si la locution Qui dit X, dit Y présente le pronom relatif sans antécédent en position frontale - tout comme de nombreux proverbes, la structure n'est pas équivalente aux moules proverbiaux en qui. La locution étudiée ici contient nécessairement le verbe dire dans les deux segments de celle-ci.

Le proverbe est également analysé par Anscombre (1994) pour démontrer que le sens du pronom qui équivalait jusqu'au XVII ${ }^{\mathrm{e}}$ siècle à si on.

2

Remarquons au passage que le pronom relatif qui en position frontale sans antécédent fait toujours référence à de

l'animé, raison pour laquelle il est paraphrasable par tout homme qui, si l'on ou celui qui.

Le proverbe est également analysé par Anscombre (1994) pour démontrer que le sens du pronom qui équivalait jusqu'au XVII ${ }^{\mathrm{e}}$ siècle à si on. 
Le point commun avec les proverbes consiste par conséquent en la présence du pronom relatif sans antécédent en position frontale et en la lecture générique que concède entre autres le pronom.

Parmi les 275 exemples de notre corpus, 1,5\% correspondent au français préclassique (de 1500 à 1630/1650), 25\% au français classique (de 1630/1650 à 1789), 40\% au français de la fin du XVIII (à partir de 1789) et du XIX ${ }^{\mathrm{e}}$ siècles, et $33 \%$ au français des $\mathrm{XX}^{\mathrm{e}}$ et $\mathrm{XXI}^{\mathrm{e}}$ siècles.

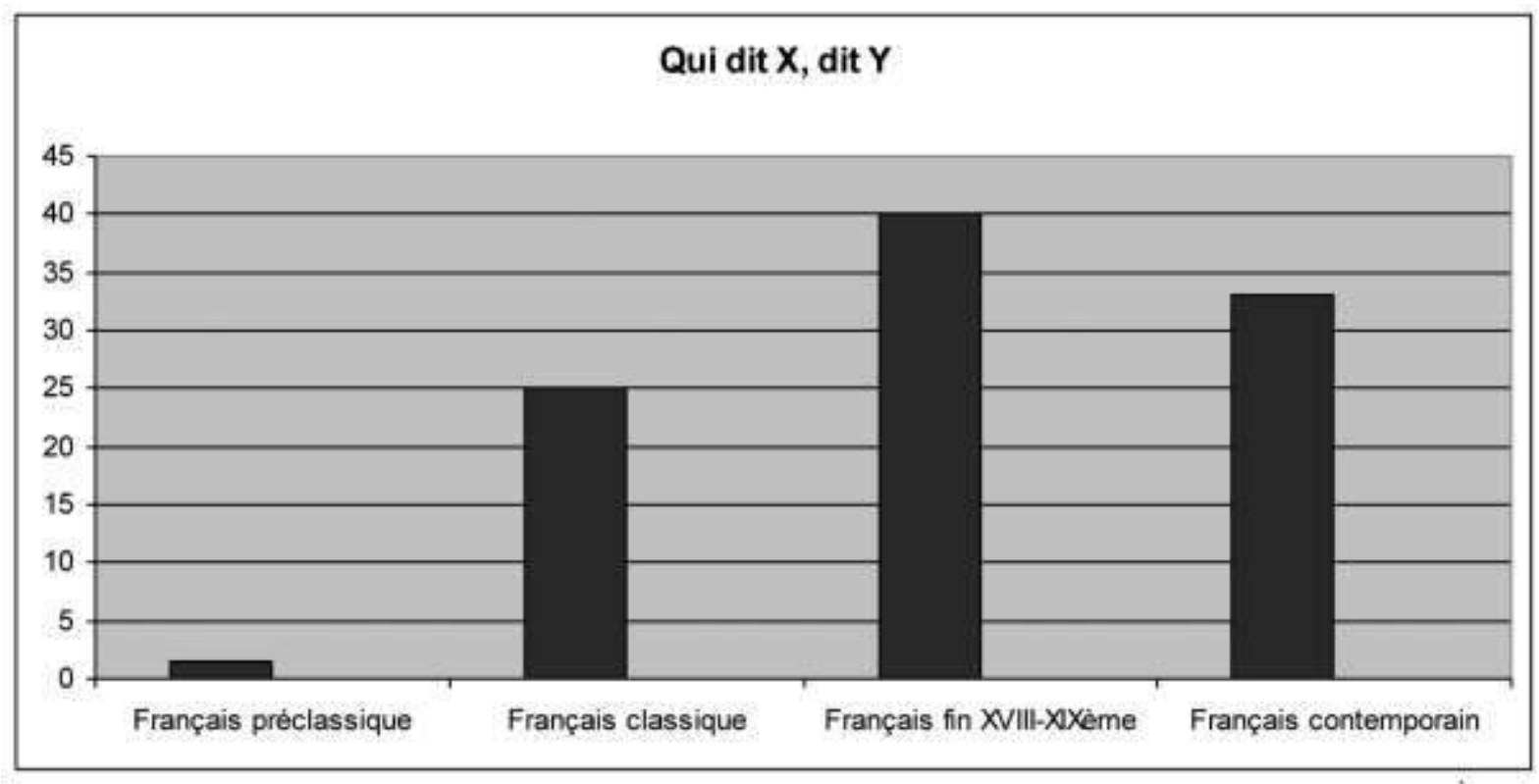

En français classique $\left(1630 / 1650\right.$ - 1789) et jusqu'à la première moitié du XIX ${ }^{\mathrm{e}}$, la structure de la locution n'est pas figée et les structures sont complexes.

La locution se présente en français préclassique et classique principalement sous les structures suivantes, présentées en ordre décroissant de fréquence. Nous nous limitons aux principales structures, sans souci d'exhaustivité.

Qui dit substantif à article zéro $(\varnothing \mathrm{N})$, dit substantif à article zéro $(\varnothing \mathrm{N})$ :

(5) Nous avons véritablement des démonstrations, mais des démonstrations de foi ; et qui dit foi, dit lumiére et ténébres.

Jacques Abbadie, Traité de la vérité de la religion chrétienne, 1684, p. 359.

Bien qu'il s'agisse de la structure la plus fréquente en français préclassique et classique, il faut signaler qu'elle ne constitue que $10 \%$ des occurrences trouvées entre le XVI et le XXI ${ }^{\mathrm{e}}$ siècles. Ainsi, $10 \%$ des occurrences correspondent au français préclassique et classique, mais 50\% des exemples proviennent du xix ${ }^{e}$ et $40 \%$ du XX ${ }^{e}$ et $X X X I^{e}$ siècles. En effet, de nos jours, il s'agit de la structure par excellence de cette locution - Qui dit argent, dit dépenses (Stromae, Alors on danse).

Qui dit adjectif, dit proposition subordonnée relative :

(6) (...) de mers, de monts, de riuieres

il luy borne ses frontieres,

et luy dit, tenez vous là, 
car si vous passez cela, si vous franchissez vos bornes, je vous abbatray les cornes. Bref, qui dit grand icy bas, dit ce qui ne dure pas : mesme la machine ronde, qu'on appelle icy le monde, vn iour se des-vnissant, et se des-arrondissant par vne affreuse tempeste, s'en ira cu par sur teste.

Georges De Brébeuf, Lucain travesti, ou les Guerres civiles de César et de Pompée, 1656, p. 44.

$$
\text { Qui dit adjectif/ article Ø N/pronom, dit pronom (tout/l'autre) : }
$$

(7) Le martyre est l'abrégé de toutes les vertus : qui dit martyr, dit tout ; et qui a donné sa vie, a consommé le sacrifice d'holocauste dont la bonne odeur monte jusqu'à Dieu.

Fénélon, Sermons et entretiens sur divers sujets, 1706, p. 279.

(8) Lisette : Madame la Marquise est si peu disposée à se marier, qu'elle ne veut même pas voir d'amants : elle m'a dit de vous prier de ne pas vous obstiner à l'aimer.

Le comte : Non plus qu'à la voir, sans doute?

Lisette : Mais je crois que cela revient au même.

Lubin : Oui, qui dit l'un dit l'autre.

Marivaux, La Seconde surprise de l'amour, 1728, p.716.

Remarquons que cette structure, qui figure toujours avec les pronoms tout ou l'autre, n'apparait dans le corpus qu'en français préclassique et classique.

Qui dit GN indéfini, dit proposition subordonnée relative (parfois suivie d'une autre relative juxtaposée) :

(9) Qui dit un rapporteur dit un homme qui s'offre pour faire ce métier, qui s'insinue par cet horrible métier, et qui par conséquent est manifestement indigne de toute croyance.

Fénélon, Examen de conscience sur les devoirs de la royauté, 1702, p.119.

Qui dit GN indéfini, dit GN indéfini

(à nouveau la locution Qui dit X, dit Y s'enchaîne sur la même locution) :

(10) Quel est le grand secret du ciel, si ce n'est cette éternelle et impénétrable communication entre le père, le fils et le Saint-Esprit ? C'est là, dis-je, le secret du ciel, qui rend heureux ceux qui le voyent et qui n'avoit point encore esté parfaitement révélé ; mais Jésus-Christ nous le révèle ici d'une manière admirable.

Qui dit un père, dit un fils; et qui dit un fils, dit un égal dans la nature; et qui dit un égal dans une nature aussi parfaite que celle de Dieu, dit un égal en toute perfection, en sorte qu'il n'y puisse avoir de premier et de second, que par une sainte, parfaite et éternelle origine.

Jacques-Bénigne Bossuet, Méditations sur l'Évangile, 1704, p. 625. 
Comme nous le verrons par la suite, l'article indéfini n'apparaît pratiquement plus en français contemporain, la structure prototypique étant celle à article zéro, comme dans Qui dit mariage, dit enfants.

Qui dit (article $\varnothing \mathrm{N}$ ), dit groupe nominal suivi de proposition subordonnée relative :

(11) Damis.

S'ils ne sont pas à plaindre, ils se plaignent toujours,

Du moins ; et, jour et nuit, voilà de leurs discours.

Pasquin.

Qui dit père, en effet, dit un homme qui gronde.

On est bien malheureux d'être fils en ce monde !

Il faut, vous soutînt-on que trois et trois font sept,

N'en pas disconvenir, et garder le tacet.

Alexis Piron, L'École des pères, 1729, p. 66.

Qui dit GN a vec expansion, dit GN avec expansion :

(12) Je connois cette sorte de respect que certains maris perfides ont pour leurs femmes, et dont ils ont l'audace et la lâcheté de se faire honneur. Qui dit aujourd'hui une femme respectée, dit une infortunée trop décente pour se plaindre de certains torts, et qui se respecte assez elle-même pour dévorer ses chagrins.

Charles Duclos, Mémoires pour servir à l'histoire des moeurs du XVIII siècle, 1751, p. 113.

Qui dit adjectif, dit adjectif :

(13) Amy quand tu viens voir Caliste,

tu luy parois toujours content ;

cependant il est très-constant,

que qui dit a moureux, dit triste.

Prends donc un air plus serieux,

fais voir ton amour dans tes yeux ; car tant que l'on te verra rire,

on ne croira jamais que tu desire.

Roger De Bussy-Rabutin, Les Mémoires de messire Roger de Rabutin, comte de Bussy, 1696, p. 7.

Qui dit article $\varnothing \mathrm{N}$, dit adjectif :

(14) Aussi quand tu fus obligeante,

Ou quand j'ay souffert tes mespris,

Ta main ne m'a jamais surpris ;

Qui dit fortune, dit changeante ;

Et j'estois tousjours preparé

à perdre un bien mal asseuré.

Georges De Scudéry, L'Amour tirannique, 1640, p. 88.

Qui dit article $\varnothing \mathrm{N}$, dit GN : 
(15) Mais l'intelligence elle-même, qui est-elle ? Qui dit intelligence, dit essentiellement la connoissance de quelque vérité. Le pur néant ne sauroit être l'objet de l'intelligence ; on ne le conçoit point ; on n'en a point d'idée ; il ne peut se présenter à l'esprit.

Fénélon, Traité de l'existence et des attributs de Dieu, 1713, p. 157.

Qui dit GN indéfini, dit GN/groupe adverbial avec expansion :

(16) Qui dit un père, dit un fils ; et qui dit un fils, dit un égal dans la nature ; et qui dit un égal dans une nature aussi parfaite que celle de Dieu, dit un égal en toute perfection, en sorte qu'il n'y puisse avoir de premier et de second, que par une sainte, parfaite et éternelle origine. Jacques-Bénigne Bossuet, Méditations sur l'Évangile, 1704, p. 625.

Qui dit GN avec expansion, dit GN avec proposition subordonnée relative (parfois avec réitération de la structure où Qui dit X, dit Yjuxtaposée) :

(17) (...) or un nombre ne peut être infini lorsqu'il peut recevoir quelque addition, et qu'on peut lui assigner une borne, du côté où il peut recevoir un accroissement. C'est même dans l'infini que mon esprit connoît le fini. Qui dit un homme malade, dit un homme qui n'a pas la santé ; qui dit un homme foible, dit un homme qui manque de force. On ne conçoit la maladie, qui n'est qu'une privation de la santé, qu'en se représentant la santé même comme un bien réel dont cet homme est privé : on ne conçoit la foiblesse, qu'en se représentant la force comme un avantage réel que cet homme n'a pas : on ne conçoit les ténèbres, qui ne sont rien de positif, qu'en niant, et par conséquent en concevant la lumière du jour qui est très-réelle et très-positive.

Fénélon, Traité de l'existence et des attributs de Dieu, 1713, p.79

Qui dit GN défini, dit GN défini :

(18) Il faut être bien aveugle, pour ne pas sentir que tout ce qui devient le caractere d'une nation, est fondé sur la nature ; et qui dit la nature, dit la raison par excellence : nous sommes ce que nous sommes, parce que de toute autre maniere nous serions plus mal.

Nicolas Rétif de la Bretonne, Le Paysan perverti ou les Dangers de la ville, 1776, p. 92.

\section{Qui dit article $\varnothing N$, dit $G N$ avec énumération d'adjectifs ou de substantifs :}

(19) Il ne nous avouoit pas, ce que nous savions déjà, que, dans ce pays là, qui dit moine, dit un homme puissant, absolu, fier, indépendant ; un homme craint des grands, respecté et presque adoré du peuple, qui n'a ni l'esprit ni la hardiesse de se scandaliser de sa conduite.

Alain-René Lesage, Aventures du chevalier de Beauchêne, 1732, p. 361.

Qui dit nom propre, dit pronom :

(20) Car afin que l'on le sache,

C'est Sillery qui s'attache

A vouloir que de nouveau,

Sire Loup, Sire Corbeau Chez moi se parlent en rime.

Qui dit Sillery, dit tout ;

Peu de gens en leur estime

Lui refusent le haut bout.

La Fontaine, Tircis et Amarante, Fable VIII, 13, 1674. 
Le but de cette énumération de structures n'est autre que de vérifier le nombre de variantes possibles en français préclassique et classique, alors qu'à partir du $\mathrm{XIX}^{\mathrm{e}}$ siècle celles-ci se réduiront considérablement. La locution possède par conséquent une liberté dans la forme absente en français contemporain. Ce qui varie principalement au sein de ces structures est le déterminant. L'article zéro est le déterminant le plus important - qui dit foi, dit lumiére et ténébres (exemple 5), suivi de l'indéfini singulier - qui dit un père, dit un fils (exemple 16) - puis du défini singulier - qui dit la nature, dit la raison par excellence (exemple 18). Les trois déterminants ont une valeur générique dans la locution, mais ce ne sera que l'article zéro qui l'emportera en français contemporain. Outre l'énumération de ces structures, nous devons signaler plusieurs caractéristiques de la locution en français préclassique et classique. D'une part, la longueur de la locution. En effet, au cours des siècles celle-ci se raccourcit pour obtenir aux $\mathrm{XX}^{\mathrm{e}}-\mathrm{XIX}^{\mathrm{e}}$ siècles une formule brève comme Qui dit argent, dit dépenses. En revanche, le français préclassique et classique présente le plus souvent une locution très longue, juxtaposant même parfois la même locution répétée :

(21) Qui dit nombre, dit amas d'unités réellement distinguées, et réciproquement indépendantes les unes des autres pour exister et n'exister pas. Qui dit amas d'unités réciproquement indépendantes, dit un tout qu'on peut diminuer, et qui par conséquent n'est point infini. Fénelon, Traité de l'existence et des attributs de Dieu, 1713, p.197.

(22) Qui dit un père, dit un fils ; et qui dit un fils, dit un égal dans la nature ; et qui dit un égal dans une nature aussi parfaite que celle de Dieu, dit un égal en toute perfection, en sorte qu'il n'y puisse avoir de premier et de second, que par une sainte, parfaite et éternelle origine.

Jacques-Bénigne Bossuet, Méditations sur l'Évangile, 1704, p.625.

(23) Je connois cette sorte de respect que certains maris perfides ont pour leurs femmes, et dont ils ont l'audace et la lâcheté de se faire honneur. Qui dit aujourd'hui une femme respectée, dit une infortunée trop décente pour se plaindre de certains torts, et qui se respecte assez elle-même pour dévorer ses chagrins.

Charles Duclos, Mémoires pour servir à l'histoire des moeurs du XVIII siècle, 1751, p.113.

La longueur de la structure ne fait que démontrer la flexibilité que possédait la locution, ce qui est encore visible dans l'introduction de certains adverbes à l'intérieur de la formule :

(24) On croit ordinairement que dans une république les desseins sont digerés avec plus de profondeur, et conduits avec plus de sagesse que sous un autre gouvernement. Qui dit cependant le conseil d'un état libre, dit un conseil tumultueux, où il y a plus de cabales et de partis que dans celui d'un roi, sans qu'il s'y trouve une puissance supérieure qui tempere l'égalité.

Abbé De Mably, Parallèle des Romains et des François par rapport au gouvernement, 1740, p.223.

À ce stade de la langue française, la locution n'est pas figée, alors qu'en français contemporain elle ne présente pas cette variété de formes possibles. Nous vérifions à nouveau le non figement de la locution en français classique dans un exemple comme celui-ci :

(25) Mais qui dit une loi dans un état libre dit une chose devant laquelle tout citoyen tremble, et le roi tout le premier.

Jean-Jacques Rousseau, Considérations sur le gouvernement de Pologne, 1771, p.1002

D'ailleurs, si en français contemporain les segments X et Y sont interchangeables - Qui dit madeleine, dit Proust / Qui dit Proust, dit madeleine ; Qui dit camembert, dit Normandie / Qui dit Normandie, dit camembert - il n'en était pas ainsi en français préclassique et classique. En effet, le segment X est le 
plus souvent bref en français préclassique et classique, et c'est $Y$ qui est long. Nous verrons dans la partie consacrée à la sémantique que la locution en français préclassique et classique véhicule le plus souvent une définition de $\mathrm{X}, \mathrm{X}$ étant alors le segment à définir et $\mathrm{Y}$ la définition.

(26) Qui dit suffisant dit tout ce qui est nécessaire pour agir ; et il servirait de peu aux dominicains de s'écrier qu'ils prennent en un autre sens le mot de suffisant : le peuple, accoutumé à l'intelligence commune de ce terme, n'écouterait pas seulement leur explication.

Blaise Pascal, Les Provinciales, 1657, p.24.

(27) Qui dit intelligence, dit essentiellement la connoissance de quelque vérité.

Fénélon, Traité de l'existence et des attributs de Dieu, 1713, p.157.

Un autre phénomène qui apparaît dans le corpus du français classique et préclassique est la juxtaposition de substantifs ou d'adjectifs dans le segment Y.

(28) Il ne nous avouoit pas, ce que nous savions déjà, que, dans ce pays- là, qui dit moine, dit un homme puissant, absolu, fier, indépendant ; un homme craint des grands, respecté et presque adoré du peuple, qui n'a ni l'esprit ni la hardiesse de se scandaliser de sa conduite.

Alain-René Lesage, Aventures du chevalier de Beauchêne, 1732, p. 361.

(29) Qui dit un caractere, dit un mêlange, dit un composé de plusieurs défauts et de plusieurs vertus, dans lequel mêlange certain vice domine si le caractere est vicieux ; c'est une vertu laquelle y domine si le caractere doit être vertueux.

Abbé Jean-Baptiste Dubos, Réflexions critiques sur la poésie et la peinture, 1733, p.231.

1.2. Propriétés syntaxiques de la locution en français contemporain :

du XIX ${ }^{e}$ au XXI ${ }^{e}$ siècle

À partir de 1807, les structures commencent à se réduire et les groupes nominaux et substantifs de la locution seront presque toujours précédés de l'article zéro. Cependant, il s'agit encore d'une étape de transition qui semble se figer dans la deuxième moitié du XIX , à partir de 1846, d'après les occurrences consultées dans Frantext. Dans la première moitié du XIX ${ }^{\mathrm{e}}$ siècle, il est encore possible de trouver quelques occurrences où $\mathrm{Y}$ est un GN indéfini :

(30) Qui dit pamphlet, dit un écrit tout plein de poison

P. L. Courrier, Pamphlet des pamphlets, 1824

(31) Qui dit toilette, en parlant des femmes, dit un grand mot, vous le savez

Alfred de Musset, Le chandelier, 1835

Même si elles sont rares, il existe également des structures avec un article défini, telle que :

(32) Qui dit la prose, dit la forme moins figurée et la moins concrète

Jules Michelet, Introduction à l'histoire universelle, 1831

La plupart des occurrences de la première moitié du XIX ${ }^{\mathrm{e}}$ se présentent sous la forme Qui dit article $\varnothing$ $\mathrm{N}$, dit article $\emptyset \mathrm{N}$ :

(33) Qui dit art, dit mensonge

Honoré de Balzac, La Peau de chagrin, 1831

ou en moindre mesure, sous la forme, Qui dit article $\varnothing \mathrm{N}$, dit article $\varnothing$ GN plus expansion : 
(34) Qui dit égoïsme, dit profond calcul

Honoré de Balzac, Histoire de la grandeur et la décadence de César Birotteau, 1837.

À partir de la deuxième moitié du XIX ${ }^{\mathrm{e}}$ siècle, le seul article qui semble pouvoir être présent dans la locution est l'article zéro ${ }^{4}$. La structure la plus représentative et que vérifie environ la moitié des occurrences est celle en Qui dit article $\varnothing \mathrm{n}$, dit article $\varnothing \mathrm{N}$ :

(35) Qui dit examen, dit révolte

Honoré de Balzac, Sur Catherine de Médicis, 1846

(36) Qui dit mendiant, dit espion

Victor Hugo, Quatre-vingt-treize, 1874

Il y a aussi de nombreuses structures en Qui dit article $\emptyset \mathrm{N}$, dit article $\varnothing \mathrm{GN}$ plus expansion, structure qui comme nous l'avons vu, existait dans une moindre mesure au début du XIX ${ }^{\mathrm{e}}$ siècle.

(37) Qui dit guerre, dit terrain sans limite

Maréchal Foch, Des principes de la guerre, 1911

Les structures en Qui dit article $\varnothing \mathrm{GN}$ plus expansion, dit article $\varnothing \mathrm{GN}$ plus expansion sont également présentes dans le corpus, quoique en moindre mesure (environ $9 \%$ du corpus à partir de 1846) :

(38) Qui dit grand penseur, dit penseur bienfaisant

Victor Hugo, William Shakespeare, 1864

Les structures en Qui dit adjectif, dit adjectif sont également représentatives :

(39) Qui dit puritain, dit méchant Victor Hugo, Choses vues, 1885

(40) Qui dit jeune, dit seul Paul Eluard, La Rose publique, 1934

Finalement, une structure qui n'apparaît qu'au $\mathrm{xx}^{\mathrm{e}}$ siècle est celle en Qui dit article $\varnothing \mathrm{N}$, dit nom propre :

(41) Qui dit jeux-vidéos, dit Super Mario, (TV5 monde. www.tv5.org. 7 jours sur la planète, février 2012).

(42) Qui dit madeleine, dit Proust,

Gérard Genette, Bardadrac, 2006

(43) Qui dit surréalisme, dit André Breton.

(samedi 30 janvier 2012, TF1)

L'article zéro s'est imposé dans les structures du français contemporain - Qui dit gonzesse, dit complications / Qui dit sainteté, dit actions vertueuses. L'article zéro, joue un rôle dans la lecture générique de la locution. Très présent également dans les structures proverbiales, - Mariage sous la pluie dure toute une vie / Chien qui aboie ne mord pas / Jeux de mains, jeux de vilains - l'article zéro apporte un certain type de généricité. Suivant les termes de Kleiber (1989), nous parlerons d'une généricité homogène, où le substantif précédé de l'article zéro se présente comme un syntagme de

$4 \quad$ Les transitions se font de façon graduelle et parmi les 125 exemples de la locution trouvés dans Frantext entre 1846 et le $x^{\mathrm{e}}$ siècle, il y a juste deux occurrences avec l'article défini et un exemple avec l'article indéfini. 
masse où il n'y aurait pas différents $\mathrm{X}$ possibles, mais un $\mathrm{X}$ représentant de tous les $\mathrm{X}$, de façon homogène, comme lorsque l'on parle de l'eau ou du sable. Dans Qui dit gonzesse, dit complications, le locuteur parle de la gonzesse comme représentative de toutes les gonzesses, sans faire de différences entre les brunes, les blondes, les grandes ou les petites.

Anscombre (1990 : 122) montre à ce propos la relation existant entre l'homogénéité, les cadres discursifs et l'article zéro : «[...] la condition d'homogénéité est en fait propre aux expressions introduisant un espace discursif, et confirme donc indirectement le lien entre les espaces discursifs et le déterminant zéro. ». En effet, comme nous le verrons avec les exemples en contexte, la locution pose un cadre de discours présenté comme étant déjà connu et admis par l'interlocuteur.

\section{PROPRIÉTES SÉMANTIQUES DE LA LOCUTION QUI DIT X, DIT Y: STÉRÉOTYPES, GÉNÉRICITÉ ET PROPRIÉTES INTRINSEQUES}

\subsection{Propriétés sémantiques en français préclassique et classique}

La relation entre les segments $\mathrm{X}$ et $\mathrm{Y}$ de la locution Qui dit $\mathrm{X}$, dit $\mathrm{Y}$ est une relation stéréotypique et dénote une généricité typifiante a priori. Pour cela, nous rappellerons brièvement les différents types de phrases génériques ainsi que les différents stéréotypes, d'après la théorie développée par JeanClaude Anscombre depuis les années 1990.

Les phrases génériques peuvent être divisées en trois types, selon les études de Kleiber (1987, 1988 ou 1989 entre autres) et d'Anscombre (2001). On distingue généralement les phrases génériques nécessairement vraies ou analytiques, du type de Les baleines sont des mammifères, les phrases génériques généralement vraies (ou typifiantes a priori), comme Les singes mangent des bananes, puis les phrases génériques synthétiques (ou locales), du type de Les Italiens sont sympathiques. Les analytiques sont les seules à ne pas admettre d'exceptions. Les phrases typifiantes a priori sont présentées comme véhiculant l'opinion d'un ON-énonciateur, d'une communauté linguistique. Elles se présentent comme étant ON-Vraies, vraies pour la communauté linguistique. Dans notre communauté linguistique Les singes mangent des bananes est présenté comme étant admis, que ce soit vrai ou non dans le monde réel. Quant aux locales, Les Italiens sont sympathiques, elles sont L-Vraies, vraies pour le locuteur, dans la mesure où elles véhiculent l'avis du locuteur.

Quant aux stéréotypes, d'après la définition proposée dans Anscombre (2001 : 58), nous appellerons stéréotype attaché à un terme une suite ouverte de phrases attachées à ce mot, chaque phrase étant pour le terme considéré une phrase stéréotypique.

Il distingue deux types de stéréotypes, le stéréotype primaire, qui est associé de façon stable au mot, au sein d'une communauté linguistique - singe-banane - et le stéréotype secondaire, attaché localement à l'occurrence d'un terme, Italien- sympathique. Ce qui est à rapprocher des différentes phrases génériques. Dans les phrases stéréotypiques secondaires, le locuteur émet une opinion qui lui est propre, en revanche une phrase stéréotypique primaire est présentée comme étant vraie pour toute une communauté linguistique. 
Revenons à nos locutions. Quand le locuteur énonce une locution en Qui dit X, dit Y, il présente $\mathrm{Y}$ comme un stéréotype primaire de X. Sous X-Y il y aurait une phrase générique typifiante a priori ${ }^{5}$.

(44) Damis.

S'ils ne sont pas à plaindre, ils se plaignent toujours,

Du moins ; et, jour et nuit, voilà de leurs discours.

Pasquin.

Qui dit père, en effet, dit un homme qui gronde.

On est bien malheureux d'être fils en ce monde!

Il faut, vous soutînt-on que trois et trois font sept,

$\mathrm{N}$ 'en pas disconvenir, et garder le tacet.

Valère.

Oui. Qu'un démêlé naisse entre un fils et son père,

Le père suit sa fougue, et le fils se modère :

Leur droit n'est toutefois que le droit du plus fort.

Alexis Piron, L'École des Pères, 1729, p.66.

Dans cette occurrence, gronder est présenté comme un stéréotype primaire de père, et il y aurait sous cette relation entre $\mathrm{X}$ et $\mathrm{Y}$ une phrase générique telle que Les pères sont des personnes qui grondent. Il s'agirait d'une des phrases stéréotypiques attachées à père. Qui plus est, $\mathrm{Y}$ est présenté comme étant une propriété intrinsèque essentielle de X. Nous rappellerons brièvement, suivant Anscombre (2001 : 65) « (...) qu'une propriété $\mathrm{P}$ est intrinsèque de I si elle est constitutive de I. Sinon, elle est extrinsèque. » Il ajoute : «Les propriétés intrinsèques essentielles d'une classe sont représentées par les phrases génériques analytiques ou typifiantes a priori. » Dans ce cas, gronder est présenté comme une propriété intrinsèque essentielle de père. De la même façon dans :

(45) Amy quand tu viens voir Caliste, tu luy parois toujours content ; cependant il est tresconstant, que qui dit amoureux, dit triste. Prends donc un air plus serieux, fais voir ton amour dans tes yeux ; car tant que l'on te verra rire, on ne croira jamais que tu desire.

Roger De Bussy-Rabutin, Les Mémoires de messire Roger de Rabutin, comte de Bussy, 1696, p.77.

Triste est présenté comme une propriété intrinsèque essentielle de amoureux. D'ailleurs, selon Anscombre $(2001: 65):$ « [...] seule une propriété intrinsèque accidentelle peut être prédite d'un SN spécifique, jamais une propriété essentielle », ce qu'il démontre par le biais des exemples suivants :

(46) ?? Ce nordique est blond.

(47) Cet homme a un seul rein.

Dans nos exemples, le locuteur en insérant père/homme qui gronde au sein de la locution, présente $\mathrm{Y}$ comme propriété intrinsèque essentielle de $\mathrm{X}$, et nous ne pourrions pas avoir dans ce contexte :

(48) ?? Ce père est un homme qui gronde.

Cela se voit très clairement dans les cas où $\mathrm{Y}$ est présenté comme une définition de $\mathrm{X}$. En effet, nous remarquons en français classique que de nombreuses occurrences véhiculent en $\mathrm{Y}$ une définition de $\mathrm{X}$, ce qui ne sera plus le cas en français contemporain :

\footnotetext{
$5 \quad$ Même si tous les stéréotypes primaires ne correspondent pas nécessairement à des phrases génériques et nous
} pourrons trouver également des phrases événementielles (voir Anscombre 2001). 
(49) C'est même dans l'infini que mon esprit connoît le fini. Qui dit un homme malade, dit un homme qui n'a pas la santé; qui dit un homme foible, dit un homme qui manque de force.

Fénelon, Traité de l'existence et des attributs de Dieu, 1713, p.79.

Ne pas avoir la santé est une propriété intrinsèque essentielle, et définitoire, de homme malade, de la même façon que manquer de force est une propriété intrinsèque essentielle de homme faible et l'on ne pourrait pas avoir :

(50) ?? Cet homme malade n'a pas la santé.

(51) ?? Cet homme faible manque de force.

De nombreuses occurrences du français classique présentent $\mathrm{Y}$ comme une définition de $\mathrm{X}$ :

(52) Qui dit l'infini, dit quelque chose qui comprend tout sans exception.

Fénelon, Traité de l'existence et des attributs de Dieu, 1713, p.115.

(53) Il ne nous avouoit pas, ce que nous savions déjà, que, dans ce pays là, qui dit moine, dit un homme puissant, absolu, fier, indépendant ; un homme craint des grands, respecté et presque adoré du peuple, qui n'a ni l'esprit ni la hardiesse de se scandaliser de sa conduite.

Alain-René Lesage, Aventures du chevalier de Beauchêne, 1732, p. 361.

Reprenant les occurrences ci-dessus, nous pouvons gloser la locution Qui dit X, dit Y par X est Y, ce qui vient à l'appui d'une glose définitoire de $\mathrm{x}$ en $\mathrm{Y}$ :

(54) Qui dit un homme malade, dit un homme qui n'a pas la santé.

Un homme malade est un homme qui n'a pas la santé.

(55) Qui dit un homme faible, dit un homme qui manque de force.

Un homme faible est un homme qui manque de force.

(56) Qui dit l'infini, dit quelque chose qui comprend tout sans exception.

L'infini c'est quelque chose qui comprend tout sans exception.

(57) Qui dit moine dit un homme puissant, absolu, fier, indépendant.

Les moines sont des hommes puissants, absolus, fiers, indépendants.

Dans ce type d'occurrences où $\mathrm{Y}$ est présenté comme une définition de $\mathrm{X}$, d'une part $\mathrm{Y}$ est généralement un segment plus long que X. D'autre part, $\mathrm{X}$ et $\mathrm{Y}$ ne sont pas interchangeables. Dans Qui dit moine, dit un homme puissant, absolu, fier, indépendant, $\mathrm{X}$ est plus bref que $\mathrm{Y}$ et les deux segments ne sont pas interchangeables. La locution n'est pas nécessairement l'équivalent de Les hommes puissants, absolus, fiers, indépendants sont des moines.

Steuckardt (2003: 231-2) rappelle à propos des définitions introduites par c'est-à-dire au XVIII ${ }^{\mathrm{e}}$ siècle que : "Quand elle est perçue comme une équivalence de signification, la glose en c'est-à-dire n'est pas sans évoquer la définition lexicographique. Elle en rappelle la forme, car, généralement, le terme Y est plus développé que le terme X. Cela ne surprendra pas : la glose est suscitée par le besoin d'expliquer X, de déployer son sens. [...] Ces « définitions » intratextuelles sont évidemment moins complètes qu'une définition lexicographique. La glose définitionnelle en discours ne donne qu'un des équivalents possibles du mot, celui qui est pertinent pour les énonciateurs. " C'est ce que nous vérifions par exemple dans Qui dit un homme malade, dit un homme qui n'a pas la santé, où Y est une glose qui définit et qui explique $\mathrm{X}$, d'où sa longueur par rapport à $\mathrm{X}$. 
2.2. Propriétés sémantiques en français contemporain :

du XIX ${ }^{\mathrm{e}}$ au XXI ${ }^{\mathrm{e}}$ siècles

Le sens contemporain de la locution commence à apparaître à partir de la deuxième moitié du xix ${ }^{\mathrm{e}}$ siècle. La locution ne véhicule plus une définition de $\mathrm{X}$. Les éléments $\mathrm{X}$ et $\mathrm{Y}$ sont maintenant interchangeables. Finalement, aussi bien le segment X que Y sont brefs. Voici un petit échantillon d'occurrences de la locution entre le $\mathrm{XIX}^{\mathrm{e}}$ et le $\mathrm{XXI}^{\mathrm{e}}$ siècles :

(58) Qui dit André Breton dit surréalisme, qui dit surréalisme dit André Breton. (TF1, samedi 30 janvier 2012.)

(59) Ils sont tous là : héros de péplums ou de contes de fées, créatures fantastiques, tous héros de jeux vidéos et qui dit jeux vidéos, dit Super Mario. Le célèbre plombier a déjà 25 ans, toujours plus agile, et, en version 2009, on peut y jouer en famille : à deux, trois ou quatre.

(TV5Monde. www.tv5.org. 7 jours sur la planète, février 2012.)

(60) [...] la cafetière de Balzac, la pomme de newton, la langue d'Einstein, le pinson sur le fusil de Darwin, la chambre de liège, la petite madeleine de Proust et «son » questionnaire (j'ai d'ailleurs entendu mentionner un jour « le questionnaire de la madeleine de Proust »; non seulement qui dit madeleine dit Proust, mais de plus en plus qui dit Proust dit madeleine), la salopette de Butor et celle de Coluche, les bretelles de Larry King, les jets de Karajan, les moteurs de Michelangeli, les loups d'Hélène Grimaud, les lentilles de Spinoza, la promenade quotidienne de Kant au sortir de sa sieste dogmatique [...].

Gérard Genette, Bardadrac, 2006, p. 222.

(61) Et il arrêtait pas de me dire : «Une fille, Jean-Claude, il me faut une fille ! » ça revenait de plus en plus souvent, comme un tube qui monte au hit-parade. "Négatif ! je lui répondais pour changer. On est en train de se faire oublier, c'est pas pour replonger dans les emmerdes. Qui dit gonzesse dit complications, tu devrais le savoir. Si on tend la main à la société, elle nous lâchera plus, jusqu'à ce qu'on soit à l'ombre. Ça te tente ? Personnellement je suis pas partant. » Bertrand Blier, Les Valseuses, 1972, p. 120.

(62) L'homme a été le problème du dix-huitième siècle ; la femme est le problème du dixneuvième. Et qui dit la femme, dit l'enfant, c'est-à-dire l'avenir. La question ainsi posée apparaît dans toute sa profondeur.

Victor Hugo, Actes et Paroles 3, 1876, p. 854.

Ces exemples de Qui dit X, dit Y permettent de voir qu'entre $\mathrm{x}$ et $\mathrm{Y}$ il y a une relation forte, comme si l'un n'allait pas sans l'autre. La femme est attachée à l'enfant, gonzesse est attaché à complications, Proust est attaché à madeleine dans une relation stéréotypique. Deux gloses sont possibles. Qui dit X, dit $\mathrm{Y}$, équivaut à Quand on parle de $\mathrm{X}$, on pense tout de suite à $\mathrm{Y}$ :

(63) Quand on parle de madeleine, on pense tout de suite à Proust.

(64) Quand on parle de la femme, on pense tout de suite à l'enfant.

(65) Quand on parle de gonzesse, on pense tout de suite à complications. 
Sous cette glose, le ON-énonciateur ${ }^{6}$ transparaît sous le on de on parle, on pense. Remarquons au passage que ce $\mathrm{ON}$ est déjà sous-jacent dans le pronom relatif qui sans antécédent ${ }^{7}$.

Si nous prenons l'occurrence suivante, provenant d'un article sur les huiles pour le corps, nous trouvons une certaine similarité sémantique avec la locution :

(66) «Quand on parle d'huile, on pense nutrition, donc peau sèche. Et on a raison: c'est un produit génial pour compenser les agressions quotidiennes qui dessèchent fortement l'épiderme (Huile Dior Prestige...). », (Elle, août 2012, p.46)

Il aurait été possible d'avoir à la place : Qui dit huile, dit nutrition, donc peau sèche... Une autre glose possible serait Dire $X$ c'est comme si on disait $Y$ :

(67) Dire la femme, c'est comme si on disait l'enfant

(68) Dire madeleine, c'est comme si on disait Proust

(69) Dire gonzesse c'est comme si on disait complications

Dans tous les cas, il existe une relation stéréotypique entre X et Y. Par exemple, sous madeleine (X) il y a une phrase stéréoypique, appartenant à notre communauté linguistique, telle que la madeleine relève du monde proustien (Y).

Quand le locuteur énonce Qui dit gonzesse, dit complications ou Qui dit madeleine, dit Proust, il n'y a pas directement allusion à la parole d'un autre. Le locuteur, en employant la locution, fait écho à un stéréotype qui est présenté comme étant admis de l'interlocuteur, puisqu'il provient d'un ONénonciateur, d'une communauté linguistique dont le locuteur fait partie. Les faits que le locuteur rapporte lui parviennent de façon médiate parce que la situation décrite - la relation entre madeleine et Proust par exemple - relève de la connaissance admise ou transmise par la tradition. Tout le monde sait, dans notre communauté linguistique, que madeleine est attaché à Proust, ou en tout cas, le locuteur en insérant madeleine-Proust sous la locution Qui dit X, dit Y, présente cette relation comme connue de l'interlocuteur, qu'elle le soit ou non. Le locuteur en énonçant Qui dit X, dit Y indique que l'information transmise n'a pas été créée par lui-même, mais qu'elle provient d'un ON-énonciateur.

Le locuteur coule sous la locution Qui dit X, dit $\mathrm{Y}$, un stéréotype primaire ou une phrase générique typifiante a priori, ou plutôt il présente la relation entre $\mathrm{X}$ et $\mathrm{Y}$ comme étant un stéréotype primaire. Le fait de couler X-Y dans cette locution crée, déclenche un stéréotype primaire. Même si la relation $\mathrm{X}$-Y peut être un stéréotype secondaire, une opinion du seul locuteur, par exemple Italienne- décolleté, dans Qui dit Italienne, dit décolleté, le fait de couler X-Y dans la locution Qui dit X, dit Y, fait que le locuteur présente cette relation comme si elle était admise de toute une communauté linguistique et non seulement de lui.

\footnotetext{
6 Nous signalons, à la suite d'Anscombre (2005), qu'il est préférable de parler d'un ON-Énonciateur, et non d'un ON-Locuteur, sachant qu'il s'agit de l'opinion de la communauté linguistique, d'un point de vue. Ceci correspond à l'énonciateur, dans la théorie de la polyphonie, et non au locuteur qui ne représente pas un point de vue, mais un personnage linguistique responsable de l'énonciation hic et nunc d'un énoncé donné.

$7 \quad$ Faute de place, nous ne pouvons nous attarder sur le pronom qui. Nous signalerons juste, suivant l'étude de Bertrand (2003), que qui pouvait être interprété en moyen français et jusqu'en français classique comme si on. Déjà signalé dès Anscombre (1994), comme à l'origine de nombreuses formes sentencieuses.
} 
Nous irons plus loin, et nous dirons que le locuteur en employant Qui dit X, dit Y, fait l'opération suivante :

Parmi toutes les propriétés attachées à $\mathrm{X}$, il en dégage une, $\mathrm{Y}$, qu'il présente comme étant la propriété la plus représentative, la propriété prototypique de X.

Ainsi, en disant :

(70) Qui dit Picasso, dit cubisme

le locuteur, parmi toutes les propriétés de Picasso (Picasso-Latin-Lover / Picasso-Folie / PicassoFemmes / Picasso-époque Bleue / Picasso-Peintre / Picasso-Montmartre / Picasso-Cubisme), dégage une de ces propriétés, dans ce cas cubisme, qu'il présente comme étant la propriété prototypique de Picasso.

De même, le locuteur en disant :

(71) Qui dit gonzesse, dit complications

dégage une des propriétés de gonzesse, dans ce cas complications, et il présente complications comme étant la propriété prototypique des gonzesses, que ceci soit vrai ou non dans le monde réel. Le locuteur présente $\mathrm{Y}$ comme prototypique de $\mathrm{X}$ et la relation $\mathrm{X}$-Y comme étant une relation connue et admise de notre interlocuteur, puisque provenant de notre communauté linguistique. Il ne s'agit plus, comme en français préclassique et classique d'une relation définitionnelle entre $\mathrm{X}$ et $\mathrm{Y}$ où $\mathrm{X}$ apparaissait comme un élément plus court que $\mathrm{Y}$ et $\mathrm{Y}$ comme une définition de $\mathrm{X}$.

Dans cette dernière occurrence, $\mathrm{Y}$ est clairement montré comme étant intrinsèque à $\mathrm{X}$ :

(72) Or, le perfectionnement de l'homme n'est-il pas lié au perfectionnement de l'humanité ? Qui dit homme dit humanité. L'homme porte en lui l'humanité. L'homme n'existe pas indépendamment de l'humanité.

Pierre Leroux, De l'humanité, de son principe et de son avenir, 1840, $\mathrm{p}$.

\section{POLYPHONIE DE LA LOCUTION}

En outre, Qui dit X, dit Y est une locution polyphonique dans la mesure où le locuteur introduit un point de vue $-\mathrm{Y}$ attaché à $\mathrm{X}$ - comme provenant d'un énonciateur collectif, d'une communauté linguistique. Le locuteur donne toujours son accord à ce point de vue. Ceci est souligné lorsque la locution est introduite par des verbes comme savoir ou par des introducteurs tels que Il est très constant que. L'énonciation de la locution est similaire dans son fonctionnement sémantique à celle des proverbes et des phrases génériques. En effet, comme dans l'énonciation proverbiale, nous pouvons trouver la locution et son contraire :

(73) Esope.

Pouvez-vous ne rien croire et dormir d'un bon somme?

De la vie à la mort il s'agit d'un instant ;

Et que peut-on risquer qui soit plus important?

Qui dit dieux, dit vengeurs ; et leur foudre...

Iphicrate.

Au contraire :

Qui dit dieux, dit clémens. 
Un remords bien sincère

Arrête, en expirant,

Leur foudre prête à choir.

Edme Boursault, Ésope à la cour, 1702, p.165.

Nous pourrions parler d'une joute de stéréotypes. Ici le locuteur énonce un premier stéréotype où au terme dieux est attachée la phrase les dieux sont vengeurs. La vengeance est présentée comme une propriété intrinsèque essentielle de dieux. Son interlocuteur lui présente un stéréotype contraire, où au terme dieux serait attachée la phrase stéréotypique les dieux sont cléments, présentant cette fois-ci la clémence comme une propriété intrinsèque essentielle de dieux. Ceci montre que les locuteurs possèdent un stock de stéréotypes attachés au même terme, se situant dans un cadre de discours ou un autre. Dans cette occurrence, la situation devient polémique, étant donné que les deux locuteurs se placent dans des cadres de discours contraires.

Ce qui est remarquable dans cette locution, c'est que le simple fait de couler deux segments dans la structure Qui dit X, dit Y, montre la relation stéréotypique entre X-Y comme étant admise de toute la communauté linguistique. Ainsi dans l'exemple suivant :

(74) Que la franchise est rare en ce siecle où nous sommes !

La feinte seulement est la vertu des hommes,

Sur tout l'art de tromper est frequent à la Cour;

Qui dit un Courtisan, dit un fourbe en amour.

L'un pour se divertir se fait une Maistresse;

L'autre fait le galant pour monstrer son adresse;

L'un par coustume agit, l'autre par interest;

Enfin tous sont Amans, et si pas un ne l'est.

Tristan L’Hermite, La Célimène : comédie de Rotrou, 1653, p.578.

$\mathrm{Y}$, un fourbe en amour, est présenté comme un stéréotype primaire de $\mathrm{X}$, un Courtisan, et comme une propriété intrinsèque essentielle de X. Il serait possible de gloser la locution par Les Courtisans sont fourbes en amour. Or cela ne veut pas dire que les courtisans soient réellement fourbes en amour. Cependant le fait de «couler» X-Y - courtisans/fourbes en amour - dans la locution Qui dit X, dit Y, rend leur relation comme étant stéréotypique. Le locuteur présente la relation entre $\mathrm{X}$ et $\mathrm{Y}$ comme un point de vue qui ne lui appartient pas, il provient de sa communauté linguistique, et il lui donne son accord. Ce point de vue est présenté comme étant On-Vrai et doit par conséquent être admis de son interlocuteur.

(75) - Vous n'aimez pas les marsouins?

- Nous les haïssons.

- Pour quelle raison les haïssez-vous?

-Vous le demandez? Les marsouins ne sont-ils pas les voisins des pingouins?

- Sans doute.

- Eh bien ! C'est pour cela que les pingouins haïssent les marsouins.

- Est-ce une raison?

- Certainement. Qui dit voisins dit ennemis. Voyez le champ qui touche au mien. C'est celui de l'homme que je hais le plus au monde.

Anatole France, L'Île des pingouins, 1908, p. 10.

Ici, de même, en énonçant Qui dit voisins dit ennemis, le locuteur met en scène un énonciateur, sa communauté linguistique, d'après laquelle la caractéristique la plus prototypique des voisins est celle d'être ennemis. Il est intrinsèque aux voisins d'être ennemis. Le locuteur donne son accord à ce $\mathrm{ON}$ énonciateur, et en fait partie, d'où le fait de pouvoir introduire la locution par On sait que : On sait que qui dit voisins dit ennemis. 


\section{CONCLUSION}

L'analyse des propriétés syntaxiques et sémantiques de la locution permet de vérifier une évolution en diachronie du français préclassique au français contemporain. La structure de la locution évolue le long des siècles de même que le sens de celle-ci. Ainsi, nous passons de structures longues et variées en français préclassique et classique qui se réduisent visiblement en français contemporain, et ce dès le $\mathrm{XIX}^{\mathrm{e}}$ siècle. De même, le sens de la locution n'est pas tout à fait le même en français classique et en français contemporain. Dans le premier cas, la locution véhicule très souvent une relation de définition entre $\mathrm{X}$ et $\mathrm{Y}$, alors qu'en français contemporain la relation entre les deux segments relève plutôt des stéréotypes et plus particulièrement des prototypes.

Sonia GÓMEZ-JORDANA FERARY Universidad Complutense de Madrid 


\section{BIBLIOGRAPHIE}

Dictionnaires et bases de données

L'Atelier historique de la langue française. L'histoire des mots du haut Moyen âge au XIX $\mathrm{e}^{\mathrm{e}}$ siècle (1999), MARSANE, éd. Redon. Contient Oudin, A. (1640), Curiositez Françoises pour supplément aux dictionnaires ou recueil de plusieurs belles proprietez, avec une infinité de proverbes et quolibets, pour l'explication de toutes sortes de Livres.

FURETIERE, A. (1690), Dictionnaire Universel, contenant generalement tous les mots françois tant vieux que modernes.

Dictionnaire de l'Académie française (1762).

VOLTAIRE (1765), Dictionnaire philosophique et compléments.

GuIzot, F. (1822), Dictionnaire universel des synonymes de la langue française.

LITTRE, E. (1872), Dictionnaire de la langue française.

La Curne De SaInTe-PalaYe, J. B. (1876), Dictionnaire historique de l'ancien langage François.

NiCOT, J., $1621=1960$, Thresor de la langue francoise tant ancienne que moderne (...) avec une grammaire françoyse et latine, et le recueil des vieux proverbes de la France, Paris, (eds.) A. et J. Picard et C*.

REY, A., 2012, Dictionnaire historique de la langue française, Paris, Le Robert.

www.frantext.fr, développé par le CNRS-ATILF (Analyse et traitement informatique de la langue française) et l’Université de Nancy2.

Études

ANSCOMBRE, J. C., 1990, « Thème, espaces discursifs et représentations événementielles », dans

Fonctionnalisme et pragmatique, J.-C. Anscombre et G. Zaccaria (éds), Milan, Ediozioni Unicopoli, p. 43150 .

ANSCOMBRE, J. C., 1994, « Proverbes et formes proverbiales : valeur évidentielle et argumentative », Langue française 102, p. 95-107.

ANSCOMBRE, J. C., 2001, «Dénomination, sens et référence dans une théorie des stéréotypes nominaux», Cahiers de praxématique 36 , p. 43-72.

Anscombre, J. C., 2005, « Le ON-locuteur : une entité aux multiples visages », dans J. Bres, P.-P. Haillet, S. Mellet, H. Nølke et L. Rosier (éds), Dialogisme et polyphonie : approches linguistiques, Bruxelles, De BoeckDuculot, p. 75-94.

BERTRAND, O., 2003, «Evolution syntaxique du pronom indéfini QUI en français : une étude diachronique », Mémoire en temps advenir, Louvain, Peeters, Orbis / Supplementa, 22, p. 381-397. 
DENDAle, P. et TASMOWSKI, L. (éds), 1994, Les sources du savoir et leurs marques linguistiques, Langue française 102.

DuCROT, O., 1984, Le dire et le dit, Paris, Les Editions de minuit.

FOURNIER, N., 1998, Grammaire du français classique, Paris, Belin.

GómeZ-JordanA, S., 2014, «Qui dit études, dit travail. Médiativité, modalité et polyphonie d'une locution de longue date », dans J.-C. Anscombre, E. Oppermann-Marsaux, A. Rodríguez Somolinos (éds.), Médiativité, polyphonie et modalité en français : études diachroniques et synchroniques, Paris, Presses de la Sorbonne nouvelle, p.101-117.

Grevisse, M., Goose, A., 1993, Le Bon Usage. Grammaire française, $13^{\mathrm{e}}$ édition rev. et augm. Paris/Louvainla-Neuve, Duculot.

Guentcheva, Z., 1996, L’énonciation médiatisée, Louvain, Paris, Peeters.

KLEIBER, G., 1987, Du côté de la référence verbale. Les phrases habituelles, Berne, Peter Lang.

KLEIBER, G., 1988, « Phrases génériques et raisonnement par défaut », Le Français moderne 56, 1/2, p. 1-15.

KLEIBER, G., 1989, L’article LE générique. La généricité sur le mode massif, Genève, Librairie Droz.

LARdon, S. et Thomine, M-C, 2009, Grammaire du français de la Renaissance, Paris, Classiques Garnier.

MENARD, P., 1994, Syntaxe de l'ancien français, Bordeaux, Bière.

RIEGEL, M., 1987, « «Qui dort dîne» ou le pivot implicatif dans les énoncés parémiques », Travaux de linguistique et de littérature XXIV, 1, p. 85-99.

Riegel, M., Pellat, J.C., Rioul, R., 1994, Grammaire méthodique du français, Paris, PUF.

SPILlebOUT, G., 1985, Grammaire de la langue française du XVII ${ }^{\mathrm{e}}$ siècle, Paris, Picard.

SteucKardT, A., 2003, « C'est- à- dire au XVIII siècle », dans Le mot et sa glose, A. Steuckardt et A. NiklasSalminen (dirs.), Publications de l'Université de Provence, p. 223-244. 